\title{
Preliminary Conception of Art Education Upgrading Medical Postgraduates' Humanistic Quality*
}

\author{
Yanan Zheng \\ Department of Psychology \\ Gannan Medical University \\ Ganzhou, Jiangxi, China 341000 \\ Rural Medical Education Research Center \\ Gannan Medical University \\ Ganzhou, Jiangxi, China 341000
}

\author{
Zhihua Zhang \\ Department of Psychology \\ Gannan Medical University \\ Ganzhou, Jiangxi, China 341000
}

\author{
Xi Gong \\ Rural Medical Education Research Center \\ Gannan Medical University \\ Ganzhou, Jiangxi, China 341000
}

\begin{abstract}
Currently a tendency can be found that the training of humanistic quality is neglected in medical postgraduate education across China, and art education plays a better role in teaching in the training of humanistic quality of medical postgraduates. This article mainly introduces the significance of art education in the medical humanistic education of medical postgraduates in Chinese medical colleges and universities and raises a basic plan to carry out the art education in order to provide references for the training of humanistic quality of medical postgraduates and teaching reform of humanistic capabilities in colleges and universities in the future.
\end{abstract}

Keywords-postgraduate education; medical postgraduate; humanistic quality; art education

\section{INTRODUCTION}

Going with the rapid development of modern medical science, conversion of medical models, changes of traditional disease spectrum and improvement of medical technologies, the society has had new requirements for medical education modes. The typical characteristic is that medical education pays more and more attentions to the humanistic quality of medical students [1]. However, influenced by the long-term biomedical models in China, the medical education still tends to "Stress clinical, neglect humanities, learn about disease knowledge in class but not practice" [2-3], researches concerned show, due to the long term neglect of humanistic education in medical colleges, the humanistic quality of medical students in the country is lower, which does no good to the development of medical and health care [4-5]. Compared to medical undergraduates, medical postgraduates are required to complete training in clinic and scientific

*Fund project: Topic of Medical Education Study (2016B-RW048) by Society of Medical Education, CMA, Special Committee of Medical Education of China Association of Higher Education in 2016; 2016 Study Program of Degree and Postgraduate Education and Teaching Reform in Jiangxi Province (jxyjg2016155). research within three years. In the face of pressures such as heavy scientific research tasks, clinic skill training as well as employment and further, how to incubate clinic skills and scientific research abilities and make students be full of humanistic feelings within a limited time have been a significant topic for medical postgraduate education in the future[6]. Considering the status quo of medical humanistic education and teaching practice, the article proposes a preliminary conception and plan where during the medical postgraduate education, the art education and practice can be used to promote the humanistic quality so as to provide references for the reform of medical humanistic education and professional ethical consciousness of medical postgraduates in the future.

\section{SignifiCANCE OF ART EDUCATION IN THE HUMANISTIC EdUCATION OF MEDICAL PostgraduATES}

\section{A. Make Medical Postgraduates Pay More Attentions to the} Humanistic Spirit and Create A New Way for the Medical Humanistic Education

Compared to theoretical courses such as Medical Ethics, Doctor-Patient Communication, Medical Sociology, Medical Psychology, Health Law as well as clinic and bedside teaching, the humanistic medical education can be done via music appreciation, fine arts works analysis, medical literature works reading, medical scene play simulation, medical movie and TV works appreciation and doctor-patient related short movies, which may work better to save teaching time and attract the eyeballs of medical postgraduates. At the beginning of a new semester or weekend, set art courses such as medial fine arts or offer art education during the teaching of doctor-patient communication and politics and moral, and turn the dull medical humanistic courses into an art form easy to get for medial postgraduates, which will help them to care about the 
humanistic spirit and professional quality in the clinic practice, improve teaching effects and achieve the teaching aims.

\section{B. Help Medical Postgraduates for Trans-Positional Thought and Better Understand What Patients Think}

Compared to medical undergraduates, medical postgraduates should master the doctor-patient relations at a deeper level, through doctor-patient related short movies, clinic medical works, medical movie appreciation and so on, medical postgraduates may consider the doctor-patient problems from a more objective angle, especially they participate and act as patients to communicate with real patients or watch art products as "patients", for example, do not whisper before patients, family members of patients with mental blocks such as somatization disorder know nothing about the symptoms of their relatives, STD patients who feel hard to speak out, patients who suffer from cancers feel desperate to life, the art education offers medical postgraduates more objective angle to examine the current doctor-patient relations and what the patients think, it will do good to the trans-positional thought in the future and build a patientoriented professional ethics consciousness.

\section{Help to Enrich Camp Activities and Improve the Humanistic Medicine Course System for Medial Postgraduates}

Due to the characteristics of medical profession, medical students have heavy courses, especially the medical postgraduates have heavier pressure in clinic and scientific research, and many reasons have caused that campus activities in medical colleges are less than that in comprehensive universities and normal universities, which may do harm to the all-side development of medical students. The proper art education can not only promote the humanistic medical education but also improve the poor conditions that medical postgraduates have few campus activities when studying, helpful to further explore and optimize the medical postgraduate course systems.

\section{Help to Protect the Mental Health of Medical Postgraduates}

Researches show, due to the characteristics of the field, the mental health of medical students is kept at a low level [7]. Yet the art education such as music appreciation, painting appreciation, classic literature works reading can not only help improve the humanistic quality of medial postgraduates and improve the skills for communication and exchanges, but also help them release pressure during the medical practice, having an effect of art mental intervention and keeping mental health [8].

\section{PRELIMINARY CONCEPTION TO BUILD AN EDUCATIONAL SYSTEM FOR MEDICAL POSTGRADUATES}

\section{A. Improve the Medical Postgraduate Course System}

At present, most of medical postgraduate course systems are "politics and moral+ foreign language + basic courses for scientific research + professional courses + few humanistic medical courses". For the class hours are few, it is difficult to set additional art courses. During the course setting, art education can be added to the politics and moral, humanistic medical courses and clinic practice discussion, such as diseases in famous paintings, add ancient poem reading in the doctorpatient communication class, enable them to participate in the doctor-patient scene plays editing, performance, short movie recording, and add music treatment in clinic psychology course or other mental intervention plans, or open a humanistic medical salon, invite experts from other colleges for lectures, engage excellent clinic doctors to show art of communications and the like. The art education is combined with medical science, scientific research, humanistic courses or more, value the linkage between medical science and art education so as to improve the licensing abilities and medical humanistic quality of medical postgraduates.

\section{B. Faculties Arrangement}

The art education courses set for medical postgraduates are finally to improve the medical humanistic quality of medical postgraduates to support the clinic medical services, rather than simply enhance the skills in artistic appreciation. Therefore, during the concrete art education, main teachers shall mostly be clinical medical staffs, combined with some art, literature or law teachers. For example, beginning with a famous painting, the art teacher will introduce the creative background, then clinical medical workers will judge whether there are any diseases through faces of characters therein; beginning with a literary works, the literary teacher will introduce the creative background and its literary value, then the clinical medical workers will introduce its signification in the doctor-patient communication or emotional expression. Due to the differences in profession, medical colleges are expected to arrange special medical teachers for further study and get art knowledge.

\section{Expand Art Atmosphere in Campus and Create An Atmosphere of Medical Humanities}

Targeting medical postgraduates, medical colleges and universities may hold "art and culture festival", "reading corner" and so on, schools, tutor group and clinical departments can also regularly organize medical postgraduates to make discussions on relevant humanistic medicine according to clinic cases and scientific research ethnics, and stir the medical postgraduates to explore the feasibilities of integrating art to medical practice via writing, movie appreciation and more, so as to improve their attentions paid to the medical professional quality, value the humanistic spirits of their own and achieve the talent training goal.

\section{Stress the Cultivation of the Communication Skills and Teamwork Spirit of Medical Postgraduates}

Doctor-patient communication means during medical activities, doctors and patients make communications on treatment, health and related factors via different ways, led by doctors, the patients receive scientific instruction and treatment to create mutual trusts between patients and doctors in order to reach an agreement on treatment[9]; Teamwork spirits for medical students mean an ability that medical students should 
own through learning relevant courses and practice, where they coordinate and support with each other in order to achieve the common goal, which is one of the professional quality [10] needed for the clinic work in the future. Generally, the abilities of medical students to communicate and teamwork both belong to the medical humanistic spirit [11], which directly affects the coming clinical work. So during the training of medical humanistic quality of medical postgraduates, the key point shall be to train their capabilities to communicate and team work. During the art education, the scene play creation, medical micro-film shooting, reading of classical literature selection as well as clinical humanities PBL case teaching all do good to improve the capabilities of communication and teamwork of medical postgraduates. During the teaching in the future, the above contents can be added to courses such as politics and moral, case discussion and so on, for example, divide the medical postgraduates into several groups, which is required to shoot a scene play within ten minutes to reflect the ethics of clinical medical treatment, besides, the medical postgraduates of other groups are arrange to watch the play and discuss how to treat during the clinical practice so as to achieve the teaching aims.

\section{E. Value the Combination of Art Education and Clinic \\ Practice Teaching}

The training goal of medical humanistic spirits for medical postgraduates is to better and properly solve the problems arising out of the medical practice, and the clinical practice with art education integrated can make them more deeply understand the medical humanistic spirit. During a concrete practice teaching, reading can be introduced to the case discussion for references and the survey and interview-based experimental teaching will make medical postgraduates personally experience the significance of art to clinic treatment, so as to really achieve the goal of rethinking of humanistic spirit.

\section{CONCLUSION}

In a word, during the humanistic medical education in medical colleges and universities, there are some problems such as low participation of postgraduates and theoretical teaching predominating, yet to integrate art education such as music, literature and performance to the humanistic medical education of medical postgraduates is an efficient improvement plan. Though the art education has certain requirements on medical teachers in a concrete teaching, yet its operating modes and features have higher attraction to medical postgraduates, owning incomparable advantages, and it will play a significant role in the medical humanistic education in the country in the future.

\section{REFERENCES}

[1] Chen Yongxiang, Liu Hong, Researches on Cognition of Humanistic Medical Knowledge [J] Medicine \& Philosophy, 2015, 36(2A): 20-23.

[2] Chen Hua, Deng Rui, Tian Dongxia, Researches on Medical Humanistic Teaching at the Perspective of Clinician, 2013,34 (12A): 75-77,83.
[3] Zheng Yanan, $\mathrm{Hu}$ Wen, Wei Zehong, Durative Somatoform Pain Disorder- 1Case Report[J]. Chinese Journal of Pain Medicine, 2016,22 (3): 239-240.

[4] Xu Minwen, Wang Qi, Jin Rui, et al, Survey and Analysis of Undergraduates' Humanistic Care in Medical Colleges and Universities[J]. Health Vocation Education, 2016, 34 (17): 102-104.

[5] Zheng Ya'nan, Chang Xinfeng, Li Yihui, Survey on Medical Students' Knowledge about Experimental Animal Ethics [J], Medicine \& Philosophy, 2016, 37 (8A): 31-33.

[6] Wang Shouyu, Liu Mingchuan, Li Huizhen, et al, Thoughts on Humanistic Education of Clinic Medical Postgraduates [J]. Medicine \& Philosophy, 2015, 36(6A): 9-11,15.

[7] Wei Ming, Li Xinhua, Wang Weiqiong, et al, Investigation on Sleep Quality of Medical Students [J]. China Journal of Health Psychology

[8] Ying Shuyue, Preliminary Discussion Modern Value of Art Treatment at the Perspective of College Students [J] Journal of Taiyuan Urban Vocational College, 2015, 5: 79-81.

[9] Wang Jinfan, Doctor-Patient Communication [M]. Ver. 2, Beijing: People's Medical Publishing House, 2012: 1.

[10] He Huaiwei, Ji Xiangnian, Analysis of Incubating Teamwork Capabilities of Medical Students in Colleges and Universities [J]. Northwest Medical Education, 2014,22 (6): 1043-1045, 1055.

[11] Wang Yu, Tang Fei, Wang Yunfeng, et al, Contents, Methods and Ways for Cultivating Professional Quality of 8-Year Medical Students in China [J] Basic and Clinical Medicine, 2014, 34 (4): 573-576. 